\title{
FUNCTIONAL IMPLICATIONS OF FLUCTUATING ASYMMETRY AMONG ENDEMIC POPULATIONS OF GASTEROSTEUS ACULEATUS
}

\author{
by
}

\author{
C.A. BERGSTROM ${ }^{1)}$ and T.E. REIMCHEN ${ }^{2,3)}$ \\ (Department of Biology, University of Victoria, P.O. Box 3020, Victoria, B.C. Canada)
}

\begin{abstract}
Summary
Fluctuating asymmetry (FA) has been used as a measure of developmental stability across many taxa, with asymmetric individuals presumed to have reduced fitness. FA has also been suggested for use in conservation biology as a measure of the health of populations. Here we assess the suitability of these uses of FA by using a novel measure of asymmetry in the bony lateral plates of threespine stickleback (Gasterosteus aculeatus) from 60 insular and endemic freshwater populations from the Queen Charlotte Islands. The frequency of asymmetric G. aculeatus individuals among populations varied from $1 \%$ to $76 \%$ with a mean of $42 \%$. Extreme variation in the frequency of asymmetries among lateral plate positions within samples was also observed. Plates important to the structural integrity of predator defences were least asymmetric, either due to selection against asymmetry at these positions or to variation in the temporal development of the plates. These results emphasize the need for caution when interpreting differential levels of FA among traits in individuals and populations, as the differences may be due to variation in the strength or direction of selection for symmetry, and not exclusively to differences in fitness.
\end{abstract}

Keywords: Gasterosteus, fluctuating asymmetry, population-wide asymmetry, developmental instability, functional morphology, conservation.

\footnotetext{
1) E-mail address: cbergstr@uvic.ca

2) E-mail address: reimchen@uvic.ca

3) We would like to thank G.E.E. Moodie and P. Hart for helpful comments on this manuscript. Many thanks to S. Douglas for help with the collection of the samples used in this study. This research has been supported by a Natural Sciences and Engineering Council of Canada (A2354) grant awarded to T.E.R. and a King-Platt Fellowship to C.A.B. This study is in partial completion of doctoral dissertation research by C.A.B.
} 


\section{Introduction}

Quantification of the fitness of an individual is simultaneously one of the most important tasks in evolutionary ecology and one of the most difficult. Fitness itself is theoretically simple in definition, but the practical measurement of such a quality quickly becomes a highly multidimensional and complex task (Endler, 1986). There is need for a simple index of fitness that can be measured at one point in time and is relieved of the burden of a complete assessment of the selective factors in an individual's habitat. Fluctuating asymmetry (FA) has been proposed as such an index of individual fitness (Soule', 1967; Møller, 1994), as it is thought to reflect an organism's genome-wide ability to buffer against stress during development (Leary et al., 1992). FA is manifested as a population-wide pattern of asymmetry in a bilateral trait which is normally distributed around a mean of zero (Van Valen, 1962; Palmer \& Strobeck, 1986). FA is indicative of random errors in the phenotypic development of an organism in response to environmental or genetic stress (Adams \& Niswander, 1967; Gest et al., 1986; Clarke \& McKenzie, 1992; Imasheva et al., 1997; Campbell et al., 1998).

There is considerable evidence that FA is selected against both in sexually selected traits (Arcese, 1994; Møller, 1994; Swaddle \& Cuthill, 1994; Watson \& Thornhill, 1994; Hansen et al., 1999), and in traits important for locomotion (Alexander et al., 1984; Møller, 1991; Balmford et al., 1993). Symmetry in freely moving animals is conceivably the ideal state, as this allows equal between-sides efficiency in a 3-dimensional, symmetric world where the direction of stimuli is unpredictable (Bradshaw \& Rogers, 1993). The symmetry of functional traits used for locomotion or defence would presumably be selected for in a population, and result in reduced numbers of individuals asymmetric at these traits in older age classes.

In this paper we assess FA among natural isolated populations of freshwater threespine stickleback (Gasterosteus aculeatus) from the Queen Charlotte Islands in northern British Columbia, Canada. Stickleback have a defensive apparatus which is composed of a series of heritable bony lateral plates (Hagen, 1973) located in parallel on both sides of the body, as well as 2 large dorsal spines and 2 large pelvic spines (Fig. 1). Freshwater stickleback from the Queen Charlotte Islands exhibit tremendous variation in the 


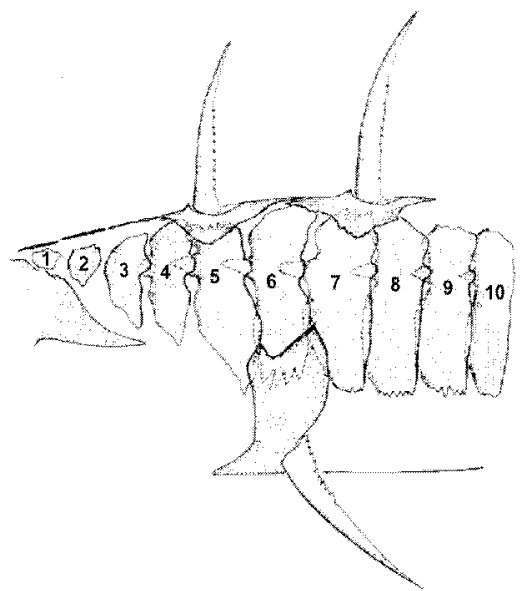

Fig. 1. Schematic of lateral plates on the anterior half of a stickleback possessing plates 1 through 10, showing areas of overlap between lateral plates and spine supports. Lateral plate positions are labelled by number. Modified from Reimchen(1983).

number of lateral plates, ranging from none to the full set of approximately 30 plates (Moodie \& Reimchen, 1976; Reimchen et al., 1985). The lateral plates are functionally important for survival; protecting the underlying integument of stickleback during predator manipulation, as well as providing structural support for the dorsal and pelvic spines (Reimchen, 1983, 1992a; review in Reimchen, 1994a).

Several studies have found asymmetries in the number of lateral plates in stickleback (Hagen, 1973; Hagen \& Gilbertson, 1973; Moodie \& Reimchen, 1976; Moodie \& Moodie, 1996), although interpretations of functional implications have been limited. Moodie \& Reimchen (1976) found a negative correlation between the degree of plate asymmetry among lakes and the presence of certain predators of stickleback, suggesting that asymmetric stickleback are at a disadvantage as prey. Here, we have greatly expanded the investigation of inter-population variation in asymmetry to include 60 populations, and have also used an additional and more rigorous index of lateral plate asymmetry.

Our first prediction is that asymmetries in the number and positions of lateral plates will compromise their effectiveness as defensive structures, therefore increasing the individual's chances of predator-mediated mortality. This will result in overall low levels of asymmetry in the lateral plates among 
natural populations of stickleback, as well as a decrease in relative frequency of asymmetric individuals with age.

Second, we will assess variation in the FA of lateral plates among 60 natural populations in order to estimate a baseline level of developmental stability for this species. Other investigators have assessed FA among populations in the wild in order to compare their relative health under varying levels of stress generated by human disturbance (Wayne et al., 1986; Clarke, 1995; Manning \& Chamberlain, 1993; Lens et al., 1999). However, it is difficult to interpret differences in FA among populations without first knowing what baseline levels of FA are found naturally (Palmer, 1996). The benefit of using populations from the Queen Charlotte Islands for this purpose is that the habitats are relatively undisturbed and pristine, therefore giving an estimate of 'natural' levels of FA with which to compare to disturbed habitats. This is the first study to our knowledge that looks at levels of FA among such a large number of wild populations.

Thirdly, we will ascertain whether there is variation in the incidence of asymmetry among lateral plate positions. The assumption that FA is an indication of overall developmental stability predicts that we should find roughly equal frequencies of asymmetry among the plate positions. However, other studies have not found high correlations in asymmetry between traits within individuals (Van Valen, 1962; Palmer \& Strobeck, 1986; Dufour \& Weatherhead, 1996). One explanation for this lack of correlation is that different traits will vary in their susceptibility to stress as a result of different developmental pathways (Møller \& Swaddle, 1997). The lateral plates of stickleback are ideal to test this prediction with, as they provide a series of structures which presumably are under similar developmental constraints and which develop in close temporal proximity of each other. Therefore, variance in developmental processes among plates should be minimal.

\section{Materials and methods}

Sampling and study area

Samples of stickleback from lakes and streams throughout the archipelago were obtained during multiple expeditions between 1975 and 1990. Habitat descriptions and general collecting methods are published elsewhere (Reimchen, 1989, 1992b, 1994b; Reimchen et al., 1985). Briefly, the majority of stickleback samples were collected from April to June using standard-mesh minnow traps placed in the littoral zones. Fish were fixed in $10 \%$ formalin and stored in $95 \%$ ethanol. Sixty of these samples were used for this study. 


\section{Morphometrics}

Morphometrics were completed on 50 sub-adults and 50 adults randomly selected from each sample, sample size allowing. Morphometrics involved the following traits: standard body length (SL), sex, position of each lateral plate on the left, position of each lateral plate on the right, total plate number on the left and total plate number on the right. Each lateral plate overlies a single myomere, and can be identified by a number (P1, P2, P3, ., P30) which is consistent between individuals (Reimchen, 1983).

Measurement error is often disguised as FA, and thus can inflate asymmetry estimates beyond what is actually present (Palmer, 1994). It is crucial to determine if measurement error is equal to, greater than or less than the FA of a trait. Therefore, after initial measurements were complete, 3 localities were randomly chosen and 20 individuals from each were remeasured for plate position. The measurement error of lateral plate position was $0.8 \%$ of the total number of plates scored on the 60 fish which were re-measured. Of the total number of lateral plates scored in the 60 samples $(N=57,487), 4.0 \%$ of them were asymmetric for position. In other words, the incidence of asymmetry was 5 times greater than that of the measurement error. The $\%$ error did not differ significantly among populations $\left(\chi^{2}=4.86\right.$; df $=2 ; 0.10>p>0.05)$. Since this study is a comparative assessment of asymmetry among populations, and the measurement error was similar among populations, we did not remove measurement error from our FA indices. If there is a difference in our FA index among populations, this will be due almost exclusively to a true FA differential since the measurement error is relatively minimal and constant among localities. All measurements were made by one individual to eliminate investigator bias.

\section{Asymmetry indices}

Asymmetry was calculated in two ways: plate number asymmetry (PNUM) and plate position asymmetry (PPOS). PNUM was calculated by subtracting the total number of plates on the left from the total number of plates on the right for each individual. This gave a signed asymmetry value for each fish ( 0 being symmetric), allowing us to test statistically for the presence of FA. We tested whether mean plate number asymmetry (PNUM) differed significantly from zero by performing a 2-tailed t-test, and found that none of the 60 populations had significant non-zero PNUM means after Bonferroni corrections. Distributions of PNUM scores for each population were examined for normality on frequency histograms. Non-normality was detected graphically in 7 of the 60 localities and were thus excluded from further analysis.

In order to calculate plate position asymmetry (PPOS), each plate along the trunk was given an asymmetry score by subtracting its presence (1) or absence ( 0 ) on the left side from the right side. Plate positions that had no plates on either side were given a null value for that position. PPOS was then calculated by summing the absolute asymmetry scores for each plate position.

Previous published studies have used PNUM as their only measure of lateral plate asymmetry in stickleback(Hagen, 1973; Hagen \& Gilbertson, 1973; Moodie \& Reimchen, 1976; Moodie \& Moodie, 1996). PPOS provides a more informative assessment of asymmetry than PNUM. In the context of this study, it allows for the inclusion of fish into the asymmetric group that have a plate present at a designated position on one side but not the other even if total plate number is symmetric (e.g. plates 2 to 7 present on the left, and 3 to 8 on the right). 
For this study we considered a fish to be asymmetric if it was asymmetric at one or more plate positions.

We compared PPOS between sexes from each locality using 2-tailed t-tests. None of the differences were significant at the $p \leqslant 0.05$ level after Bonferroni corrections. The sexes were therefore pooled for further analysis.

The relative frequency of asymmetric individuals was calculated among sampled populations. We also compared the frequency of asymmetric sub-adults and adults at each location to determine if the asymmetric individuals were being selected out of the populations. Some localities contained a mixture of stickleback with lateral plates and those that were completely naked. The validity of scoring a fish as symmetric for a trait that is absent on both sides is questionable. Twelve of the remaining 53 samples contained at least one naked stickleback, and were therefore excluded from population comparisons of frequencies of asymmetric individuals.

\section{Results}

\section{Distribution of asymmetries among populations}

The frequency of stickleback asymmetric for PNUM varied from $1 \%$ to $60 \%$ among localities $($ mean $=39 \%$ ), while the frequency of those asymmetric for PPOS varied from $1 \%$ to $76 \%$ (mean $=42 \%$; Fig. 2). As expected, the frequency of stickleback asymmetric for PNUM were in all cases equal to or less than the frequency of stickleback asymmetric for PPOS. The majority of the populations contained between $35-50 \%$ asymmetric individuals. Mean PPOS was not significantly different between sub-adults and adults for any locality at the $p \leqslant 0.05$ level after Bonferroni corrections.

\section{Distribution of asymmetries among lateral plate positions}

The frequency of asymmetric plates among the 30 possible plate positions for the pooled samples was highly variable $\left(\chi^{2}=211.41 ; \mathrm{df}=29 ; p<0.001\right.$; Fig. 3). Asymmetries occurred frequently in positions $1 \& 2$, with a sharp decrease in positions 4 to 7 followed by an increase again in positions $8 \& 9$. Posterior to position 9 there was a progressive reduction in asymmetry.

Threespine stickleback have three genetically distinct lateral plate morphologies: low plated, partially plated and completely plated (Wootton, 1984), all three of which are represented in the samples used for this study. The low-plated morph has anterior plates only, usually in the range of positions 1 to 9 , and no keel on the caudal peduncle. The completely plated 


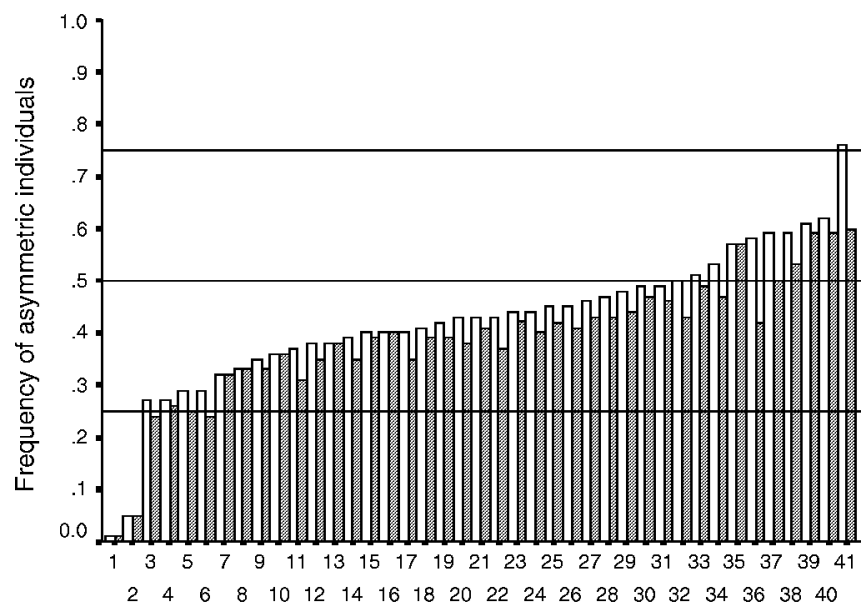

Locality

Fig. 2. Frequencies of asymmetric individuals for each sampled locality. Clear bars are frequencies of stickleback asymmetric for PPOS; shaded bars are frequencies of stickleback asymmetric for PNUM. Localities ranked according to increasing PPOS asymmetry. Localities with samples containing at least 1 plateless stickleback were excluded.(Localities: $1=$ Stiu; $2=$ Gowgaia East $3=$ Darwin; $4=$ Eden $; 5=$ Downtree $6=$ Fairfax; $7=$ South Otter; 8 = Skidegate; $9=$ Anser $; 10=$ Mayer $; 11=$ Escarpment $12=$ Puffin; $13=$ Yakoun R.; 14 = Coates; 15 = Gudal; 16 = Anderson South; $17=$ Woodpile; 18 = Cumshewa; 19 = Pontoon R.; 20 = Desolate; 21 = Sangan; 22 = Snub; 23 = Cedar; 24 = Kiokathli; 25 = Lumme Swamp.; 26 = Anderson North; 27 = Whiteswan; 28 = Lutea; 29 = Seal Inlet; 30 = Krajina; 31 = Pontoon Center; 32 = Blackwater Cr.; 33 = Amber; $34=$ Geikie 3; 35 = Smith; $36=$ Clearwater; $37=$ Debris; $38=$ Drizzle; $39=$ Capeball $; 0=$ Wright; $41=$ Lower Victoria).

morph has the full set of plates extending to the anterior tip of a welldeveloped keel. The partially plated morph has the anterior plates and a keel, as well as some intermediate plates in various positions along the trunk. Asymmetries were rare in the completely plated morph $(3.5 \%$ of the individuals of this group) compared to the partially plated (75\%) and low plated $(43 \%)$ morphs. Therefore, in order to remove any confounding effects of genetic predisposition and varying susceptibility to FA among the 3 morphs, frequency of asymmetries among plate positions were evaluated for each.

The distribution of lateral plate asymmetries among positions for the low plated morphs was highly variable and significantly deviated from an equal distribution $\left(\chi^{2}=250.40 ; \mathrm{df}=8 ; p<0.001\right.$; Fig. 4a). The distribution was 


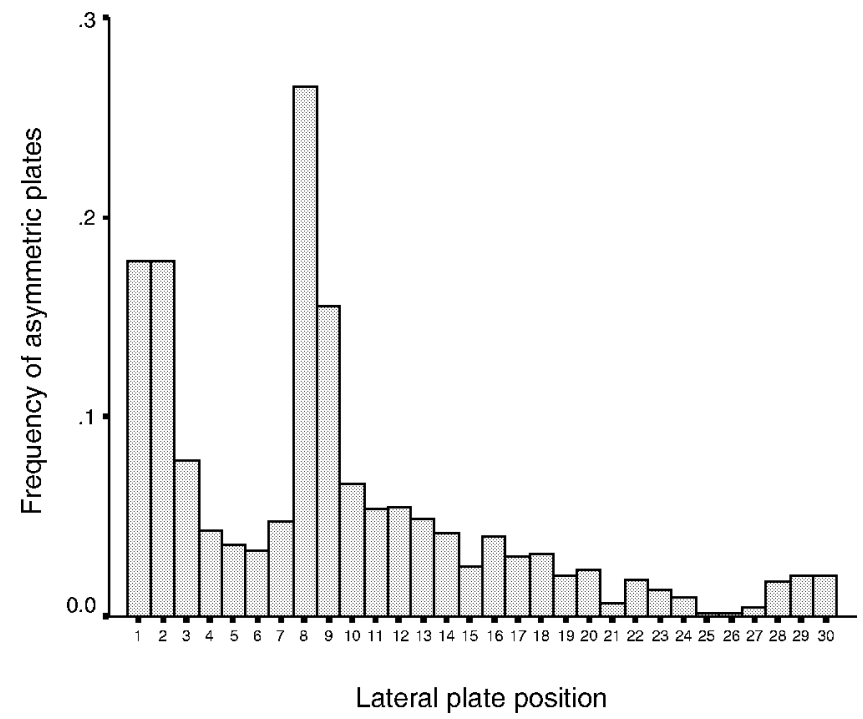

Fig. 3. Total frequencies of asymmetries among lateral plate positions for pooled locality samples. Total $N=3,702$.

U-shaped, with the most anterior and posterior positions having the highest asymmetry, and central positions 4 to 7 having the lowest.

The distribution of lateral plate asymmetries among positions for the partially plated morphs was also highly variable and significantly deviated from an equal distribution $\left(\chi^{2}=253.57\right.$; $\mathrm{df}=25 ; p<0.001$; Fig. 4b). In this group the asymmetry was greatly reduced in positions 3 to 6 , similar to the low plated morphs, as well as those positions near the caudal peduncle (24 to 26).

The completely plated morph had very low overall plate asymmetry relative to the other two morphs ( $\sim 3.5 \%$ of all completely plated individuals). The distribution of lateral plate asymmetries among positions for the complete morph was variable but did not deviate significantly from an equal distribution $\left(\chi^{2}=36.89\right.$; $\mathrm{df}=29 ; p>0.10$; Fig. 4c $)$. Asymmetries were

Fig. 4. Frequencies of asymmetries among lateral plate positions for the 3 lateral plate morphs. Locality samples are pooled. Numbers above bars denote the total number of symmetric and asymmetric stickleback at that plate position if different from the total sample size. Fish with no plates present at a position are not included in that position's sample size. Note change in $y$-axis value ranges between the three morphs. (a) Low-plated morph; total $N=3072$.

(b) Partially plated morph; total $N=233$. (c) Completely plated morph; total $N=397$. 


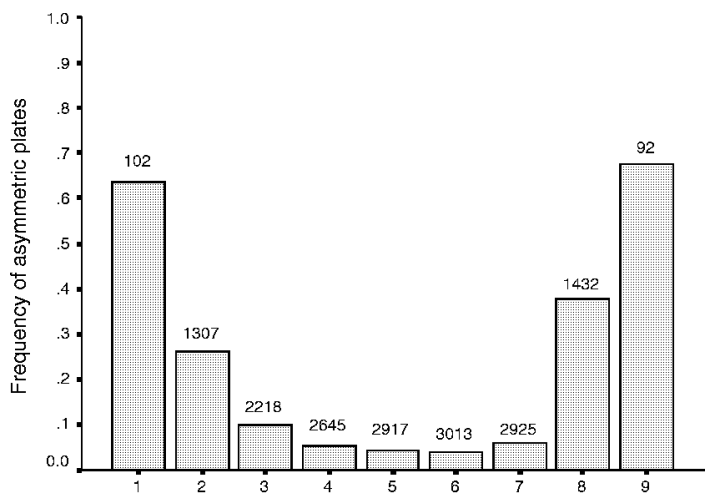

(a)

Lateral plate position

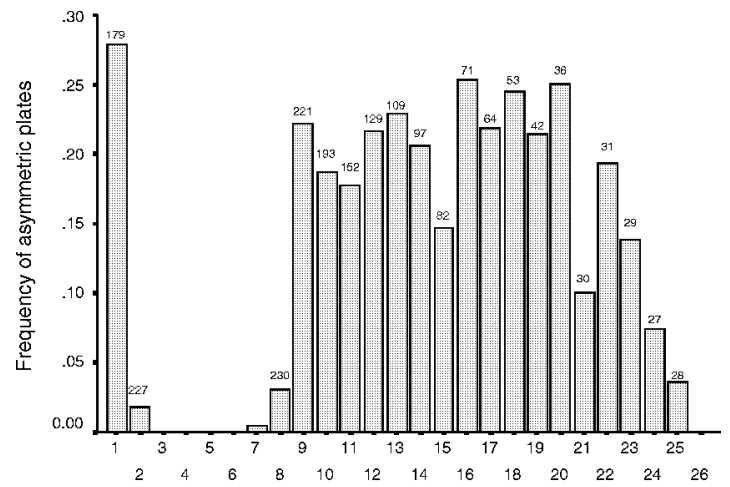

(b) Lateral plate position

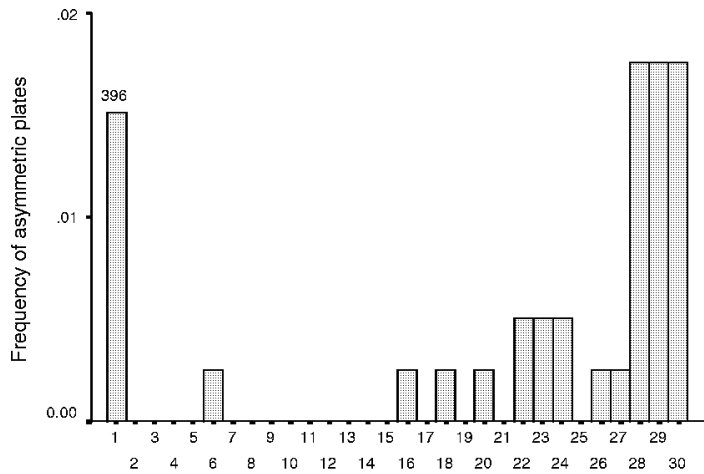

(c)

Lateral plate position 
relatively infrequent throughout plate positions (at most 7 individuals were asymmetric for a given position) and were completely absent in the majority.

\section{Discussion}

\section{Total frequency of asymmetric individuals}

Our prediction of low levels of asymmetry in the lateral plates of G. aculeatus was not supported, as the incidence of asymmetry among these natural populations was exceptionally high (average frequency was $42 \%$ ). Asymmetries in functional traits in other studies are predicted to be selected out of the gene pool (Van Valen, 1962), and in many taxa this has been demonstrated (e.g. Balmford et al., 1993; Evans et al., 1994; Swaddle, 1997). It may be that asymmetric sticklebacks in these lakes and ponds are in fact suffering increased relative mortality, but the level of asymmetry in the pre-selected sub-adults is so high each year, that the frequency of asymmetric adults remains high. However, if this were the case we would expect to see a decrease in the frequency of asymmetric individuals from sub-adults to adults, and this has not been found in any of our samples so far, or in other studies of plate number asymmetry in stickleback (Moodie \& Reimchen, 1976; Reimchen, unpubl. data).

It is possible that the asymmetry may actually provide an advantage to the stickleback, and this advantage is maintaining the high frequency of asymmetric individuals. Other studies have found subtle advantages to asymmetry in stickleback. Moodie \& Moodie (1996) found that male stickleback asymmetric for plate number were more likely to contain fry in their nests than symmetric males, and Reimchen (1997) found that juvenile stickleback asymmetric for pelvic girdle development had a significantly lower incidence of parasite infection. Asymmetry studies in other taxa as well have shown evidence that subtle asymmetries can be advantageous (e.g. Seligman, 1998). A functional advantage to asymmetry of the posterior lateral plates in stickleback may be an increase in flexibility. Stickleback perform a C-start burst acceleration when startled (Taylor \& McPhail, 1985) and a missing plate on one side may allow a greater angle to be acquired and thus a greater velocity (see below). Experiments are currently underway to investigate this further. 


\section{Distribution of asymmetries among populations}

The large variation in the frequency of asymmetric individuals among populations (Fig. 2) was unexpected, and a simple explanation for this will probably prove to be elusive. Clearly there is not a homogeneous baseline level of lateral plate asymmetry among freshwater stickleback populations. We suggest that this variation may be due to any one or more of the following factors.

First, the variation may be due to differences in the severity of natural environmental stress (temperature, $\mathrm{pH}$, productivity, inter- and intra-specific competition) among localities (e.g. Auffray et al., 1999). For example, increased competition or low productivity may result in resource depletion in gravid females. Lower allocation of energy and nutrients into the eggs may equate to increased stress during development from this maternal affect, and inflated FA in the offspring. Until a complete assessment of asymmetry in all of the samples $(\sim 180)$ is made, the possible patterns that may emerge from analyses of physical and ecological parameters of the habitats will be only suggestive.

Second, the isolated populations might vary in their genetic quality, resulting from disruption of co-adapted gene complexes via inter-population hybridization or elevated levels of homozygosity; both proposed as causes of FA (review in Møller \& Swaddle, 1997). Hybridization is unlikely between these populations, as the majority of them are geographically isolated lakes and ponds. However, some hybridization is possible with marine stickleback as a result of flooding and/or non-assortative mating between lake and stream forms, but the exact degree to which this takes place is not known. Lower Victoria Lake, which has the highest frequency of asymmetric individuals, is geographically isolated from marine waters with a high gradient stream separating them, leaving no opportunity for hybridization with marine stickleback. The inlet streams draining into Lower Victoria also have a high gradient, and thus are unlikely to have resident stream populations of stickleback, minimising the chances of hybridization between lake and stream forms. If hybridization was a primary cause of FA, one would expect to find low levels of asymmetry at this lake, contrary to our results. Levels of homozygosity in the populations have not been thoroughly assessed. A quantification of the impact these factors have on inter-population variation in FA can only be obtained with rigorous genetic analysis. 
Third, the variation may be due to differential selective pressures on asymmetric individuals among populations. Moodie \& Reimchen (1976) found a negative correlation between lateral plate number asymmetry (PNUM) and presence of predatory fish on stickleback from the Queen Charlotte Islands. It is possible that variation in predation regime or intensity among localities is generating a differential in selective pressure on asymmetric stickleback prey. A thorough analysis of variation in predation among populations is underway which includes avian predators as well as predatory fish in order to more accurately assess this relationship.

\section{Distribution of asymmetries among lateral plate positions}

The distributions of lateral plate asymmetries among the positions exhibit unexpectedly high levels of heterogeneity, contrary to our prediction. Lateral plates at positions 4 through 7 , which exhibit a drastic decrease in incidence of asymmetry, provide the major structural support for the dorsal and pelvic spines (Reimchen, 1983). There may be strong selection against asymmetry in these structural plates. The large spines on stickleback provide protection against predators (Hoogland et al., 1957) by increasing the cross-sectional diameter, often to sizes beyond the capabilities of gape-limited predators (Reimchen, 1991). If the absence of a plate on one side of a structural position equates to the spines' easy deflection during manipulation by a predator, the cost of this asymmetry may be high. This reasoning is consistent with the anterior $\mathrm{u}$-shaped distribution of asymmetries that are found in low plated and partially plated stickleback (Fig. 4a, b). These two morphs also exhibit an increase in incidence of asymmetry posterior to position 8 and anterior to position 4 . There are two possible explanations for this pattern.

The first is that with increasing distance from the structural plate positions (4 through 7), the functional cost of asymmetry decreases and therefore selection against asymmetry is reduced. Furthermore, since body angle deflection during burst $\mathrm{C}$-starts begins at mid-body (posterior to the large dorsal spines, at the anterior edge of the dorsal fin), missing plates in this region may provide an advantage via increased body flexibility. Asymmetric plate presence at these positions could allow for this increase in flexibility, while still providing the stickleback with partial integument protection from punctures during predator handling. Asymmetry at this region along the trunk might be a result of a trade-off between pre- and post-capture escape techniques, and is currently being assessed experimentally. 
The second explanation of this variation in asymmetry among positions is a developmental one. The lateral plates do not develop simultaneously at each position, but rather in series. The structural plates $5 \& 6$ develop first, followed by the more anterior and posterior plates in sequence ( 7 , then 4 , 8,3 , etc.) until development is complete (Igarashi, 1964; Bell, 1981). This happens to correspond roughly with increasing asymmetry found among the anterior plate positions. The window of susceptibility to stress during ontogeny may vary temporally, such that lateral plates developing later may be more sensitive to stress than plates that develop earlier. Clearly this issue is in need of experimental investigation.

The pattern of asymmetry among plate positions for the completely plated stickleback was statistically homogeneous, although the distribution was similar to the low and partially plated distributions (Fig. 4c). Strong selection for post-capture survival during predation events results in the maintenance of the complete morph in freshwater habitats (Reimchen, 1994a). If the post-capture benefits of complete morphs out-weigh the potential pre-capture benefits increased flexibility through asymmetry may provide, plate development in this morph may be highly canalised. In other words, if there is strong selection for the presence of a structure, there will be strong selection against developmental errors which compromise that structure's integrity. If this is the case for lateral plates in stickleback, a decrease in plate asymmetry in the complete morph, as well as in the structural plates of the low and partially plated morphs, is not surprising.

\section{Conclusions}

The results of this study suggest that the use of FA as an estimate of fitness is clearly problematic. A thorough understanding of the functional significance of and selective pressures acting on each trait in question for use in this fashion is absolutely necessary. We have demonstrated that there is high discordance of asymmetry among serial, homologous structures within and among populations, and that the structures may be under different and possibly even opposing selective forces. The accuracy of an estimate of fitness based on the FA of one or a few traits is highly dependent on the magnitude of variation of the selective pressures on the traits in question. Proceeding with a study of FA on a set of traits with less than a thorough understanding of their function will lead to questionable interpretations. 
Ideally, the assessment of asymmetry in numerous traits not subject to selection would be ideal, but the satisfactory demonstration of true neutrality is rare.

The use of FA to assess the health of a population also needs to be exercised with caution, as this study demonstrates that natural levels of FA can fluctuate drastically. Clearly more work is needed to investigate the natural causes of FA in the wild, before this measure can be trusted with confidence as an indicator of environmental stress.

\section{References}

Adams, M.S. \& Niswander, J.D. (1967). Developmental 'noise' and a congenital malformation. - Genet. Res. 10, p. 313-317.

Arcese, P. (1994). Harem size and horn symmetry in oribi. - Anim. Behav. 48, p. 14851488.

Alexander, R.McN., Brandwood, A., Currey, J.D. \& Jayes, A.S. (1984). Symmetry and precision control of strength in limb bones of birds. - J. Zool. 203, p. 135-143.

Auffray, J.C., Renaud, S., Alibert, P. \& Nevo, E. (1999). Developmental stability and adaptive radiation in the Spalax ehrenbergi superspecies in the Near-East. - J. Evol. Biol. 12, p. 207-221.

Balmford, A., Jones, I.L. \& Thomas, A.L.R. (1993). On avian asymmetry: evidence of natural selection for symmetrical tails and wings in birds. - Proc. R. Soc. Lond. B 252, p. $245-$ 251.

Bell, M.A. (1981). Lateral plate polymorphism and ontogeny of the complete plate morph of threespine sticklebacks( Gasterosteus aculeatus). - Evolution 35, p. 67-74.

Bradshaw, J. \& Rogers, L. (1993). The evolution of lateral asymmetries, language, tool use, and intellect. - Academic Press, Inc., San Diego, California, 463 pp.

Campbell, W.B., Emlen, J.M. \& Hershberger, W.K. (1998). Thermally induced chronic developmental stress in coho salmon: integrating measures of mortality, early growth, and developmental stability. — Oikos 81, p. 398-410.

Clarke, G.M. (1995). Relationships between developmental stability and fitness: application for conservation biology. - Conservation Biology 9, p. 18-24.

— — \& McKenzie, L.J. (1992). Fluctuating asymmetry as a quality control indicator for insect mass rearing processes. - J. Econ. Entomol. 8, p. 2045-2050.

Dufour, K.W. \& Weatherhead, P.J. (1996). Estimation of organism-wide asymmetry in redwinged blackbirds and its relation to studies of mate selection. — Proc. R. Soc. Lond. B 263, p. 769-775.

Endler, J.A. (1986). Natural selection in the wild. - Princeton University Press, Princeton, New Jersey, 337 pp.

Evans, M.R., Martins, T.L.F. \& Haley, M. (1994). The asymmetrical cost of tail elongation in red-billed streamertails. — Proc. R. Soc. Lond. B 256, p. 97-103.

Gest, T.R., Siegel, M.I. \& Anistranski, J. (1986). The long bones of neonatal rats stressed by cold, heat and noise exhibit increased fluctuating asymmetry. — Growth 50, p. 385-389. 
Hagen, D.W. (1973). Inheritance of numbers of lateral plates and gill rakers in Gasterosteus aculeatus. - Heredity 30, p. 303-312.

— — \& Gilbertson, L.G. (1973). Selective predation and the intensity of selection acting upon the lateral plates of threespine sticklebacks. - Heredity 30, p. 273-287.

Hansen, L.T.T., Amundsen, T. \& Forsgren, E. (1999). Symmetry: attractive not only to females. - Proc. R. Soc. Lond. B 266, p. 1235-1240.

Hoogland, R.D., Morris, D. \& Tinbergen, N. (1957). The spines of sticklebacks( Gasterosteus aculeatus and Pygosteus) as a means of defence against predators (Perca and Esox). Behaviour 10, p. 205-236.

Igarashi, K. (1964). Observation on the development of the scutes in land-locked forms of threespine stickleback, Gasterosteus aculeatus aculeatus. — Linneas. Bull. Japanese Soc. Sci. Fish. 30, p. 95-103.

Imasheva, A.G., Volker, L., Zhivotovsky, L.A. \& Lazebny, O.E. (1997). Effects of extreme temperatures on phenotypic variation and developmental stability in Drosophila melanogaster and Drosophila buzzatii. — Biol. J. Linn. Soc. 61, p. 117-126.

Leary, R.F., Allendorf, F.W. \& Knudsen, K.L. (1992). Genetic, environmental, and developmental causes of meristic variation in rainbow trout. - Acta. Zool. Fenn. 191, p. 79-95.

Lens, L., Van Dongen, S., Wilder, C.M., Brooks, T.M. \& Matthysen, E. (1999). Fluctuating asymmetry increases with habitat disturbance in seven bird species of a fragmented afrotropical forest. — Proc. R. Soc. Lond. B 266, p. 1241-1246.

Manning, J.T. \& Chamberlain, A.T. (1993). Fluctuating asymmetry in gorilla canines: a sensitive indicator of environmental stress. — Proc. R. Soc. Lond. B 252, p. 189-193.

Moodie, G.E.E. \& Moodie, P.F. (1996). Do asymmetric sticklebacks make better fathers? Proc. R. Soc. Lond. B 263, p. 535-539.

— — \& Reimchen, T.E. (1976). Phenetic variation and habitat differences in Gasterosteus populations of the Queen Charlotte Islands. - Syst. Zool. 25, p. 49-61.

Møller, A.P. (1991). Sexual ornament size and the cost of fluctuating asymmetry. — Proc. R. Soc. Lond. B 243, p. 59-62.

— - (1994). Sexual selection in the barn swallow (Hirundo rustica). IV. Patterns of fluctuating asymmetry and selection against asymmetry. — Evolution 48, p. 658-670.

— — \& Swaddle, J.P. (1997). Asymmetry, developmental stability, and evolution. — Oxford University Press, Oxford, England, 291 pp.

Palmer, A.R. (1994). Fluctuating asymmetry analysis: a primer. — In: Developmental instability: its origins and evolutionary implications (T.A. Markow, ed.). Kluwer Academic Publishers, The Netherlands, p. 335-364.

— - (1996). Waltzing with asymmetry. — BioScience 46, p. 518-532.

— — \& Strobeck, C. (1986). Fluctuating asymmetry: Measurement, Analysis, Patterns. Ann. Rev. Ecol. Syst. 17, p. 391-141.

Reimchen, T.E. (1983). Structural relationships between spines and lateral plates in threespine stickleback (Gasterosteus aculeatus). - Evolution 37, p. 931-946.

- _ (1989). Loss of nuptial colour in threespine sticklebacks (Gasterosteus aculeatus). _ Evolution 43: 450-460.

— — (1991). Trout foraging failures and the evolution of body size in stickleback. — Copeia 4, p. 1098-1104.

- — (1992a). Injuries on stickleback from a toothed predator (Oncorhynchus) and some implications for the evolution of lateral plates. — Evolution 46, p. 1224-1230. 
— - (1992b). Naikoon Provincial Park, Queen Charlotte Islands: Biophysical data for freshwater habitats. - Ministry of the Environment, Government of British Columbia, Victoria, British Columbia, $69 \mathrm{pp}$.

— - (1994a). Predators and evolution in threespine stickleback. — In: The evolutionary biology of the threespine stickleback(M.A. Bell \& S.A. Foster, eds.). Oxford University Press, Oxford, p. 240-276.

_ _ (1994b). Biophysical surveys of aquatic habitats in Gwaii Haanas 1993: Upper Victoria Lake, Lower Victoria Lake, Escarpment Lake and 14 selected streams. - Canadian Parks Service document, Queen Charlotte City, British Columbia, 176 pp.

- - (1997). Parasitism of asymmetrical pelvic phenotypes in stickleback. — Can. J. Zool. 75, p. 2084-2094.

- - Stinson E.S. \& Nelson J.S. (1985). Multivariate differentiation of parapatric and allopatric populations of threespine stickleback in the Sangan River watershed, Queen Charlotte Islands. — Can. J. Zool. 63, p. 2944-2951.

Seligman, H. (1998). Evidence that minor directional asymmetry is functional in lizard hindlimbs. - J. Zool. Lond. 245, p. 205-208.

Soule', M.E. (1967). Phenetics of natural populations. II. Asymmetry and evolution in a lizard. - Am. Nat. 101, p. 141-160.

Swaddle, J.P. (1997). Developmental stability and predation success in an insect predatorprey system. - Behav. Ecol. 8, p. 433-436.

— — \& Cuthill, I.C. (1994). Preference for symmetric males by female zebra finches. Nature 367, p. 165-166.

Taylor, E.B. \& McPhail, J.D. (1985). Prolonged and burst swimming in anadromous and freshwater threespine stickleback, Gasterosteus aculeatus. — Can. J. Zool. 64, p. 416420.

Van Valen, L. (1962). A study of fluctuating asymmetry. — Evolution 16. p. 125-142.

Watson, P.J. \& Thornhill, R. (1994). Fluctuating asymmetry and sexual selection. — Trends Ecol. Evol. 9, p. 21-25.

Wayne, R.K., Modi, W.S. \& O'Brien, S.J. (1986). Morphological variability and asymmetry in the cheetah (Acinonyx jubatus) a genetically uniform species. - Evolution 40, p. 7885.

Wootton, R.J. (1984). A functional biology of the stickleback. — University of California Press, Berkeley and Los Angeles, 265 pp. 